\title{
Transcendental structure of multiloop massless correlators and anomalous dimensions
}

\author{
P.A. Baikov ${ }^{a}$ and K.G. Chetyrkin ${ }^{b, c}$ \\ ${ }^{a}$ Skobeltsyn Institute of Nuclear Physics, Lomonosov Moscow State University, \\ 1(2), Leninskie gory, Moscow 119991, Russian Federation \\ ${ }^{b}$ Institut für Theoretische Teilchenphysik, Karlsruhe Institute of Technology (KIT), \\ Wolfgang-Gaede-Straße 1, 726128 Karlsruhe, Germany \\ ${ }^{c}$ II Institut für Theoretische Physik, Universität Hamburg, \\ Luruper Chaussee 149, 22761 Hamburg, Germany \\ E-mail: baikov@theory.sinp.msu.ru, Konstantin.Chetyrkin@kit.edu
}

ABSTRACT: We give a short account of recent advances in our understanding of the $\pi$ dependent terms in massless (Euclidean) 2-point functions as well as in generic anomalous dimensions (ADs) and $\beta$-functions. We extend the considerations of [1] by two more loops, that is for the case of 6- and 7-loop correlators and 7- and 8-loop renormalization group (RG) functions. Our predictions for the ( $\pi$-dependent terms) of the 7-loop RG functions for the case of the $O(n) \phi^{4}$ theory are in full agreement with the recent results from [2]. All available 7- and 8-loop results for QCD and the scalar $O(n) \varphi^{4}$ theory obtained within the large $N_{f}$ approach to the quantum field theory (see, e.g. [3]) are also in full agreement with our results.

KEYwords: Perturbative QCD, Renormalization Group

ARXIV EPRINT: 1908.03012 


\section{Contents}

1 Introduction $\quad 1$

2 Hatted representation: general formulation and its implications 4

$3 \pi$-structure of 3,4,5 and 6-loop p-integrals $\quad 6$

$4 \pi$-dependence of 7-loop $\beta$-functions and AD 8

$\begin{array}{lll}4.1 & \text { Tests at } 7 \text { loops } & 10\end{array}$

5 Hatted representation for 7-loop p-integrals and the $\pi^{12}$ subtlety 10

$6 \pi$-dependence of 8-loop $\beta$-functions and AD 12

$\begin{array}{lll}6.1 & \text { Tests at } 8 \text { loops } & 14\end{array}$

$\begin{array}{lll}7 & \text { Conclusion } & 15\end{array}$

\section{Introduction}

Since the seminal calculation of the Adler function at order $\alpha_{s}^{3}[4]$ it has been known that p-functions in QCD demonstrate striking regularities in terms proportional to $\pi^{2 n}$ (or, equivalently, even zetas, ${ }^{1}$ with $n$ being positive integer. Indeed, it was demonstrated in [4] for the first time a mysterious complete cancellation of all contributions proportional to $\zeta_{4} \equiv \frac{\pi^{4}}{90}$ (which generically appear in separate diagrams) while odd zetas terms (that is those proportional to $\zeta_{3}$ and $\zeta_{5}$ in the case under consideration) do survive and show up in the final result. Here by p-functions we understand ( $\overline{\mathrm{MS}}$-renormalized) Euclidean Green functions $^{2}$ or 2-point correlators or even some combination thereof, expressible in terms of massless propagator-like Feynman integrals (to be named p-integrals below).

Since then it has been noted many times that all physical (that is scale-invariant) p-functions are indeed free from even zetas at order $\alpha_{s}^{4}$ (like corrections to the Bjorken (polarized) DIS sum rule) and some of them - like the Adler function - even at the next, in fact, 5-loop, $\alpha_{e} \alpha_{s}^{4}$ order [6]. On the other hand, the first appearance of $\zeta_{4}$ in a one-scale physical quantity has been demonstrated in [7] for the case of the 5-loop scalar correlator.

It should be stressed that the limitation to QCD p-functions in the above discussion is essential. In general case scale-invariant p-functions do depend on even zetas already at 4 loops (see eq. (11.8) in [1]).

\footnotetext{
${ }^{1}$ As is well known every even power $2 n$ of $\pi$ is uniquely related to the corresponding Euler $\zeta$-function $\zeta_{2 n} \equiv \sum_{i>0} \frac{1}{i^{2 n}}$, according to a rule $\zeta_{2 n}=r(n) \pi^{2 n}$, with $r(n)$ being a (known) rational number [5].

${ }^{2}$ Like quark-quark-qluon vertex in QCD with the external gluon line carrying no momentum.
} 
To describe these regularities more precisely we need to introduce a few notations and conventions. Let

$$
F_{n}\left(a, \ell_{\mu}\right)=1+\sum_{1 \leq i \leq n}^{0 \leq j \leq i} g_{i, j}\left(\ell_{\mu}\right)^{j} a^{i}
$$

be a (renormalized) p-function in a one-charge theory with the coupling constant $a .{ }^{3}$ Here $Q$ is an (Euclidean) external momentum and $\ell_{\mu}=\ln \frac{\mu^{2}}{Q^{2}}$. The integer $n$ stands for the (maximal) power of $a$ appearing in the p-integrals contributing to $F_{n}$. In the case of onecharge gauge theory and gauge non-invariant $F$ we will always assume the case of the Landau gauge. In particularly all our generic considerations in this paper are relevant for QCD p-functions with $a=\frac{\alpha_{s}(\mu)}{4 \pi}$.

The $F$ without $n$ will stand as a shortcut for a formal series $F_{\infty}$. In terms of bare quantities $^{4}$

$$
F=Z F_{B}\left(a_{B}, \ell_{\mu}\right), \quad Z=1+\sum_{i \geq 1}^{1 \leq j \leq i} Z_{i, j} \frac{a^{i}}{\epsilon^{j}},
$$

with the bare coupling constant, the corresponding renormalization constant (RC) and AD being

$$
\begin{array}{rlrl}
a_{B} & =\mu^{2 \epsilon} Z_{a} a, & Z_{a} & =1+\sum_{i \geq 1}^{1 \leq j \leq i}\left(Z_{a}\right)_{i, j} \frac{a^{i}}{\epsilon^{j}} \\
\left(\frac{\partial}{\partial \ell_{\mu}}+\beta a \frac{\partial}{\partial a}\right) F & =\gamma F, & & \\
\gamma(a) & =\sum_{i \geq 1} \gamma_{i} a^{i}, \quad \gamma_{i}=-i Z_{i, 1} .
\end{array}
$$

The coefficients of the $\beta$-function $\beta_{i}$ are related to $Z_{a}$ in the standard way:

$$
\beta_{i}=i\left(Z_{a}\right)_{i, 1}
$$

A p-function $F$ is called scale-independent if the corresponding $\operatorname{AD} \gamma \equiv 0$. If $\gamma \neq 0$ then one can always construct a scale-invariant object from $F$ and $\gamma$, namely:

$$
F_{n+1}^{s i}\left(a, \ell_{\mu}\right)=\frac{\partial}{\partial \ell_{\mu}}(\ln F)_{n+1} \equiv\left(\frac{\left(\gamma(a)-\beta(a) a \frac{\partial}{\partial a}\right) F_{n}}{F_{n}}\right)_{n+1} .
$$

Note that $F_{n+1}^{\mathrm{si}}\left(a, \ell_{\mu}\right)$ starts from the first power of the coupling constant $a$ and is formally composed from $\mathcal{O}\left(\alpha_{s}^{n+1}\right)$ Feynman diagrams. In the same time it can be completely restored from $F_{n}$ and the $(n+1)$-loop $\mathrm{AD} \gamma$.

If not otherwise stated we will assume the so-called $G$-scheme for renormalization [8]. The scheme is natural for massless propagators. All ADs, $\beta$-functions and $Z$-factors are identical in $\overline{\mathrm{MS}}$ - and G-schemes. For (finite) renormalized functions there exists a simple conversion rule. Namely, in order to switch from an $G$-renormalized quantity to the one in

\footnotetext{
${ }^{3}$ We implicitly assume that the coupling constant $a$ counts loops.

${ }^{4}$ We assume the use of the dimensional regularization with the space-time dimension $D=4-2 \epsilon$.
} 
the $\overline{\mathrm{MS}}$-scheme one should make the following replacement in the former: $\ln \mu^{2} \rightarrow \ln \mu^{2}+2$ ( $\mu$ is the renormalization scale, the limit of $\epsilon \rightarrow 0$ is understood).

An (incomplete) list of the currently known regularities ${ }^{5}$ includes the following cases.

1. Scale-independent QCD p-functions $F_{n}$ and $F_{n}^{\mathrm{s} i}$ with $n \leq 4$ are free from $\pi$-dependent terms.

2. Scale-independent QCD p-functions $F_{5}^{\mathrm{si}}$ are free from $\pi^{6}$ and $\pi^{2}$ but do depend on $\pi^{4}$.

3. The QCD $\beta$-function starts to depend on $\pi$ at 5 loops only [14-16] via $\zeta_{4}$. In addition, there exits a remarkable identity [1]

$$
\beta_{5}^{\zeta_{4}}=\frac{9}{8} \beta_{1}^{(1)} \beta_{4}^{\zeta_{3}}, \quad \text { with } \quad F^{\zeta_{i}}=\lim _{\zeta_{i} \rightarrow 0} \frac{\partial}{\partial \zeta_{i}} F .
$$

4. If we change the $\overline{\mathrm{MS}}$-renormalization scheme as follows:

$$
a=\bar{a}\left(1+c_{1} \bar{a}+c_{2} \bar{a}^{2}+c_{3} \bar{a}^{3}+\frac{1}{3} \frac{\beta_{5}}{\beta_{1}^{(1)}} \bar{a}^{4}\right),
$$

with $c_{1}, c_{2}$ and $c_{3}$ being any rational numbers, then all known QCD functions $\hat{F}_{5}^{\mathrm{si}}\left(\bar{a}, \ell_{\mu}\right)$ and the (5-loop) QCD $\beta$-function $\bar{\beta}(\bar{a})$ both loose any dependence on $\pi$. This remarkable fact was discovered in [9] and led to the renewed interest in the issue of even zeta values in two-point correlators and related objects.

5. It should be also noted that no terms proportional to the first or second powers of $\pi$ do ever appear in all known (not necessarily QCD!) p-functions and even in separate p-integrals at least at loop number $L$ less or equal 5 . This comes straightforwardly from the fact that the corresponding master p-integrals are free from such terms. The latter has been established by explicit analytic calculations for $L=2,3$ [8], $L=4$ [17-19] and finally at $L=5[20]$. Note for the last case only a part of 5-loop master integrals was explicitly computed. However, there are generic mathematical arguments in favor of absence of contributions with weight one and two, that is $\pi$ and $\pi^{2}$ in p-integrals at least with the proper choice of the basis set of transcendental generators [21, 22]. By proper choice here we mean, essentially, a requirement that transcendental generators should be expressible in terms of rational combinations of finite p-integrals [23, 24], without use of $\pi$ as a generator.

Our results below are in full agreement with these arguments.

It should be stressed that eventually every separate diagram contributing to $F_{n}$ and $F_{n+1}^{\mathrm{si}}$ contains the following set of irrational numbers: $\zeta_{3}, \zeta_{4}, \zeta_{5}, \zeta_{6}$ and $\zeta_{7}$ for $n=4, \zeta_{3}$, $\zeta_{4}$ and $\zeta_{5}$ for $n=3$ as illustrated in table 1. Thus, the regularities listed above are quite nontrivial and for sure can not be explained by pure coincidence.

\footnotetext{
${ }^{5}$ For discussion of particular examples of $\pi$-dependent contributions into various QCD p-functions we refer to works [9-13].
} 


\begin{tabular}{|c|c||c|c|}
\hline $\mathrm{L}$ & $\mathrm{p}$-integrals & $\mathrm{L}+1$ & $\mathrm{Z}$ \\
\hline 0 & rational & 1 & rational $/ \epsilon$ \\
1 & rational $/ \epsilon$ & 2 & rational $/ \epsilon^{2}$ \\
2 & $\zeta_{3}$ & 3 & $\zeta_{3} / \epsilon$ \\
3 & $\zeta_{3} / \epsilon, \zeta_{4}, \zeta_{5}$ & 4 & $\zeta_{3} / \epsilon^{2},\left\{\zeta_{4}, \zeta_{5}\right\} / \epsilon$ \\
4 & $\zeta_{3} / \epsilon^{2},\left\{\zeta_{4}, \zeta_{5}\right\} / \epsilon, \zeta_{3}^{2}, \zeta_{6}, \zeta_{7}$ & 5 & $\zeta_{3} / \epsilon^{3},\left\{\zeta_{4}, \zeta_{5}\right\} / \epsilon^{2},\left\{\zeta_{3}^{2}, \zeta_{6}, \zeta_{7}\right\} / \epsilon$ \\
5 & $\zeta_{3} / \epsilon^{3},\left\{\zeta_{4}, \zeta_{5}\right\} / \epsilon^{2},\left\{\zeta_{3}^{2}, \zeta_{6}, \zeta_{7}\right\} / \epsilon$, & 6 & $\zeta_{3} / \epsilon^{4},\left\{\zeta_{4}, \zeta_{5}\right\} / \epsilon^{3},\left\{\zeta_{3}^{2}, \zeta_{6}, \zeta_{7}\right\} / \epsilon^{2}$, \\
& $\zeta_{3} \zeta_{4}, \zeta_{8}, \zeta_{3} \zeta_{5}, \zeta_{5,3}, \zeta_{3}^{3}, \zeta_{9}$ & & $\left\{\zeta_{3} \zeta_{4}, \zeta_{8}, \zeta_{3} \zeta_{5}, \zeta_{5,3}, \zeta_{3}^{3}, \zeta_{9}\right\} / \epsilon$ \\
\hline
\end{tabular}

Table 1. The structure of p-integrals (expanded in $\epsilon$ up to and including the constant $\epsilon^{0}$ part) and RCs in dependence on the loop number $L$. The inverse power of $\epsilon$ stands for the maximal one in generic case; in particular cases it might be less.

In this paper ${ }^{6}$ we first present a short discussion of recent advances in studying the structure of the $\pi$-dependent terms in massless (Euclidean) 2-point functions as well as in generic anomalous dimensions and $\beta$-functions. Then we extend the considerations of [1] by two more loops. Finally, we discuss remarkable connections between $\epsilon$-expansion of 4-loop p-integrals and the $D=4$ values of finite 5-, 6-, and 7-loop p-integrals.

\section{Hatted representation: general formulation and its implications}

The full understanding and a generic proof of points $1-5$ above have been recently achieved in our work [1]. The main tool of the work was the so-called "hatted" representation of transcendental objects contributing to a given set of p-integrals.

Let us reformulate the main results of [1] in an abstract form. We will call the set of all L-loop p-integrals $\mathcal{P}_{L}$ a $\pi$-safe one if the following is true.

(i) All p-integrals from the set can be expressed in terms of $(M+1)$ mutually independent (and $\epsilon$-independent) transcendental generators

$$
\mathcal{T}=\left\{t_{1}, t_{2}, \ldots, t_{M+1}\right\} \text { with } t_{M+1}=\pi .
$$

This means that any p-integral $F(\epsilon)$ from $\mathcal{P}_{L}$ can be uniquely ${ }^{7}$ presented as follows

$$
F(\epsilon)=F\left(\epsilon, t_{1}, t_{2}, \ldots, t_{M}, \pi\right)+\mathcal{O}(\epsilon),
$$

where by $F(\epsilon)$ we understand the exact value of the p-integral $F$ while the combination $\epsilon^{L} F\left(\epsilon, t_{1}, t_{2}, \ldots, t_{M}, \pi\right)$ should be a rational polynomial ${ }^{8}$ in $\epsilon, t_{1} \ldots, t_{M}, \pi$. Every such polynomial is a sum of monomials $T_{\alpha}$ of the generic form

$$
\sum_{\alpha} r_{\alpha} T_{\alpha}, \quad T_{\alpha}=\epsilon^{n} \prod_{i=1, M+1} t_{i}^{n_{i}}
$$

\footnotetext{
${ }^{6} \mathrm{~A}$ preliminary version of the present work (not including the 8-loop case) was reported on the International Seminar "Loops and Legs in Quantum Field Theory" (LL2018) in St. Goar, Germany and published in $[25]$.

${ }^{7}$ We assume that $F\left(\epsilon, t_{1}, t_{2}, \ldots, \pi\right)$ does not contain terms proportional to $\epsilon^{n}$ with $n \geq 1$.

${ }^{8}$ That is a polynomial having rational coefficients.
} 
with $n \leq L, n_{i}$ and $r_{\alpha}$ being some non-negative integers and rational numbers respectively. A monomial $T_{\alpha}$ will be called $\pi$-dependent and denoted as $T_{\pi, \alpha}$ if $n_{M+1}>0$. Note that a generator $t_{i}$ with $i \leq M$ may still include explicitly the constant $\pi$ in its definition, see below.

(ii) For every $t_{i}$ with $i \leq M$ let us define its hatted counterpart as follows:

$$
\hat{t}_{i}=t_{i}+\epsilon \sum_{\alpha} h_{i \alpha}(\epsilon) T_{\pi, \alpha}
$$

with $\left\{h_{i \alpha}\right\}$ being rational polynomials in $\epsilon$ and $T_{\pi, \alpha}$ are all $\pi$-dependent monomials as defined in (2.3). Then there should exist a choice of both a basis $\mathcal{T}$ and polynomials $\left\{h_{i \alpha}\right\}$ such that for every L-loop p-integral $F\left(\epsilon, t_{i}\right)$ the following equality holds:

$$
F\left(\epsilon, t_{1}, t_{2}, \ldots, \hat{t}_{M}, \pi\right)=F\left(\epsilon, \hat{t}_{1}, \hat{t}_{2}, \ldots, t_{M}, 0\right)+\mathcal{O}(\epsilon) .
$$

We will call $\pi$-free any rational polynomial (with possibly $\epsilon$-dependent coefficients) in $\left\{t_{i}, i=1, \ldots, M\right\}$.

As we will discuss below the sets $\mathcal{P}_{i}$ with $i=3,4,5$ are for sure $\pi$-safe (well, for $L=5$ almost) while $\mathcal{P}_{6}$ highly likely shares the property. For the case of $\mathcal{P}_{7}$ the situation is more complicated (but still not hopeless!) as discussed in section 5 . In what follows we will always assume that every (renormalized) L-loop p-function as well as ( $\mathrm{L}+1)$-loop $\beta$-functions and anomalous dimensions are all expressed in terms of the generators $t_{1}, t_{2}, \ldots, t_{M+1}$.

Moreover, for any polynomial $P\left(t_{1}, t_{2}, \ldots, \pi\right)$ we define its hatted version as

$$
\hat{P}\left(\hat{t}_{1}, \hat{t}_{2}, \ldots, \hat{t}_{M}\right):=P\left(\hat{t}_{1}, \hat{t}_{2}, \ldots, \hat{t}_{M}, 0\right) .
$$

Let $F_{L}$ is a (renormalized, with $\epsilon$ set to zero) p-function, $\gamma_{L}$ and $\beta_{L}$ are the corresponding anomalous dimension and the $\beta$-function (all taken in the $L$-loop approximation). The following statements have been proved in [1] under the condition that the set $\mathcal{P}_{L}$ is $\pi$-safe and that both the set $\mathcal{T}$ and the polynomials $\left\{h_{i \alpha}(\epsilon)\right\}$ are fixed.

\section{No- $\pi$ Theorem.}

(a) $F_{L}$ is $\pi$-free in any (massless) renormalization scheme for which corresponding $\beta$ function and $\mathrm{AD} \gamma$ are both $\pi$-free at least at the level of $L+1$ loops.

(b) The scale-invariant combination $F_{L+1}^{\mathrm{si}}$ is $\pi$-free in any (massless) renormalization scheme provided the $\beta$-function is $\pi$-independent at least at the level of $L+1$ loops.

2. $\pi$-dependence of L-loop p-functions. If $F_{L}$ is renormalized in $\overline{\mathrm{MS}}$-scheme, then all its $\pi$-dependent contributions can be expressed in terms of $\left.\hat{F}_{L}\right|_{\epsilon=0},\left.\hat{\beta}_{L-1}\right|_{\epsilon=0}$ and $\left.\hat{\gamma}_{L}\right|_{\epsilon=0}$.

3. $\boldsymbol{\pi}$-dependence of L-loop $\boldsymbol{\beta}$-functions and AD. If $\beta_{L}$ and $\gamma_{L}$ are given in the $\overline{\mathrm{MS}}$-scheme, then all their $\pi$-dependent contributions can be expressed in terms of $\left.\hat{\beta}_{L-1}\right|_{\epsilon=0}$ and $\left.\hat{\beta}_{L-1}\right|_{\epsilon=0},\left.\hat{\gamma}_{L-1}\right|_{\epsilon=0}$ correspondingly. 


\section{$3 \pi$-structure of $3,4,5$ and 6 -loop p-integrals}

A hatted representation of p-integrals is known for loop numbers $L=3$ [26], $L=4$ [17] and $L=5$ [20]. In all three cases it was constructed by looking for such a basis $\mathcal{T}$ as well as polynomials $h_{i \alpha}(\epsilon)$ (see eq. (2.4)) that eq. (2.5) would be valid for sufficiently large subset of $\mathcal{P}_{L}$.

Let us consider the next-loop level, that is $\mathcal{P}_{6}$. In principle, the strategy requires the knowledge of all (or almost all) L-loop master integrals. On the other hand, if we assume the $\pi$-safeness of the set $\mathcal{P}_{6}$ we could try to fix polynomials $h_{i \alpha}(\epsilon)$ by considering some limited subset of $\mathcal{P}_{6}$.

Actually, we do have at our disposal a subset of $\mathcal{P}_{6}$ due to work [18] where all 4-loop master integrals have been computed up to the transcendental weight 12 in their $\epsilon$-expansion. Every particular 4-loop p-integral divided by $\epsilon^{n}$ can be considered as a $(4+n)$-loop p-integral. The collection of such $(4+\mathrm{n})$-loop p-integrals form a subset of $\mathcal{P}_{4+n}$ which we will refer to as $\mathcal{P}_{4} / \epsilon^{n}$. We have tried this subset for $n=1$ and 2 .

Our results are given below. (To make resulting formulas shorter we use even zetas $\zeta_{2}=\pi^{2} / 6, \zeta_{4}=\pi^{4} / 90, \zeta_{6}=\pi^{6} / 945, \zeta_{8}=\pi^{8} / 9450$ and $\zeta_{10}=\pi^{10} / 93555$ instead of the corresponding even powers of $\pi$ ).

$$
\begin{aligned}
& \hat{\zeta}_{L=6}^{\hat{\zeta}_{3}}:=\underbrace{\zeta_{3}+\frac{3 \epsilon}{2} \zeta_{4}}_{L=3} \quad \underbrace{-\frac{5 \epsilon^{3}}{2} \zeta_{6}}_{\delta(L=4)} \underbrace{+\frac{21 \epsilon^{5}}{2} \zeta_{8}}_{\delta(L=5)} \underbrace{-\frac{153 \epsilon^{7}}{2} \zeta_{10}}_{\delta(L=6)}, \\
& \hat{\zeta}_{5=6}:=\underbrace{\zeta_{5}}_{L=3} \underbrace{+\frac{5 \epsilon}{2} \zeta_{6}}_{\delta L=4} \underbrace{-\frac{35 \epsilon^{3}}{4} \zeta_{8}}_{\delta(L=5)} \quad \underbrace{+63 \epsilon^{5} \zeta_{10}}_{\delta(L=6)}, \\
& \hat{\zeta}_{L=6}:=\underbrace{\zeta_{7}}_{L=4} \quad \underbrace{+\frac{7 \epsilon}{2} \zeta_{8}}_{\delta(L=5)} \quad \underbrace{-21 \epsilon^{3} \zeta_{10}}_{\delta(L=6)},
\end{aligned}
$$

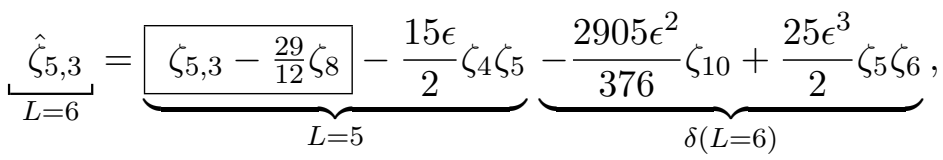

$$
\begin{aligned}
& \hat{\zeta}_{L=6}:=\underbrace{\zeta_{9}}_{L=5} \quad \underbrace{+\frac{9}{2} \epsilon \zeta_{10}}_{\delta(L=6)}, \\
& \hat{\zeta}_{L=6}:=\underbrace{\zeta_{7,3}-\frac{793}{94} \zeta_{10}+3 \epsilon\left(-7 \zeta_{4} \zeta_{7}-5 \zeta_{5} \zeta_{6}\right)}_{L=6}, \\
& \hat{\zeta}_{L=6}:=\underbrace{\zeta_{11}}_{L=6}, \\
& \hat{\zeta}_{L=6}^{\hat{\zeta}_{5,3}}:=\underbrace{\zeta_{5,3,3}+45 \zeta_{2} \zeta_{9}+3 \zeta_{4} \zeta_{7}-\frac{5}{2} \zeta_{5} \zeta_{6}}_{L=6} .
\end{aligned}
$$


Here multiple zeta values are defined as [27]

$$
\zeta_{n_{1}, n_{2}}:=\sum_{i>j>0} \frac{1}{i^{n_{1}} j^{n_{2}}}, \quad \zeta_{n_{1}, n_{2}, n_{3}}:=\sum_{i>j>k>0} \frac{1}{i^{n_{1}} j^{n_{2}} k^{n_{3}}} .
$$

Some comments on these eqs. are in order.

- The boxed entries form a set of $\pi$-independent (by definition!) generators for the cases of $L=3$ (eqs. (3.1), (3.2)), $L=4$ (eqs. (3.1)-(3.3)), $L=5$ (eqs. (3.1)-(3.5)) and $L=6$ (eqs. (3.1)-(3.8)). In what follows we will use for the boxed combinations in eqs. (3.1)-(3.8) the notation

$$
\bar{\zeta}_{n_{1}, n_{2}, \ldots}:=\left.\hat{\zeta}_{n_{1}, n_{2}, \ldots}\right|_{\epsilon=0}
$$

- There is no terms proportional to single or second powers of $\pi$ outside boxed combinations in relations (3.1)-(3.8). This fact directly leads to the absence of such terms in the (renormalized) 6-loop p-integrals and generic ADs and $\beta$-functions with the loop number not exceeding 7 . Later we will see that the same is true for 7-loop p-integrals and 8-loop RG functions (assuming the conservative scenario as described in section 5).

- For $L=5$ we recover the hatted representation for the set $\mathcal{P}_{5}$ first found in [20]. The latter coincides with eqs. (3.1)-(3.3) and (3.5) while instead of (3.4) the authors of [20] suggest

$$
\hat{\zeta}_{3,5}:=\varphi-3 \epsilon \zeta_{4} \zeta_{5}+\frac{5 \epsilon}{2} \zeta_{3} \zeta_{6}
$$

with

$$
\varphi:=\frac{3}{5} \zeta_{5,3}+\zeta_{3} \zeta_{5}-\frac{29}{20} \zeta_{8}=\zeta_{6,2}-\zeta_{3,5}
$$

Our $\hat{\zeta}_{5,3}$ (eq. (3.4)) is related (up to the corresponding order of $\epsilon$ ) to $\hat{\zeta}_{3,5}$ from [20] as $\hat{\zeta}_{5,3}=\frac{5}{3}\left(\hat{\zeta}_{3,5}-\hat{\zeta}_{3} \hat{\zeta}_{5}\right)$. The reason for this redefinition is that we want every hatted zeta to be equal to the corresponding unhatted zeta plus terms proportional to explicit powers of $\pi^{2}$ at $\epsilon^{0}$ order as well.

- We do not claim that the generators

$$
\zeta_{3}, \zeta_{5}, \zeta_{7}, \bar{\zeta}_{5,3}, \zeta_{9}, \bar{\zeta}_{7,3}, \zeta_{11}, \bar{\zeta}_{5,3,3} \text { and } \pi
$$

are sufficient to present the pole and finite parts of every 6-loop p-integral. In fact, it is not true $[2,28,29]$. However we believe that it is safe to assume that all missing irrational constants can be associated with the values of some convergent 6-loop pintegrals at $\epsilon=0$. 


\section{$4 \pi$-dependence of 7 -loop $\beta$-functions and AD}

Using the approach of [1] and the hatted representation of the irrational generators (3.13) as described by eqs. (3.1)-(3.8) we can straightforwardly predict the $\pi$-dependent terms in the $\beta$-function and the anomalous dimensions in the case of any 1-charge minimally renormalized field model at the level of 7 loops.

Our results read (the combination $F^{t_{\alpha_{1}} t_{\alpha_{2}} \ldots t_{\alpha_{n}}}$ stands for the coefficient of the monomial $\left(t_{\alpha_{1}} t_{\alpha_{2}} \ldots t_{\alpha_{n}}\right)$ in $F$; in addition, by $F^{(1)}$ we understand $F$ with every generator $t_{i}$ from $\left\{t_{1}, t_{2}, \ldots, t_{M+1}\right\}$ set to zero).

$$
\begin{aligned}
& \gamma_{4}^{\zeta_{4}}=-\frac{1}{2} \gamma_{1}^{(1)} \beta_{3}^{\zeta_{3}}+\frac{3}{2} \gamma_{3}^{\zeta_{3}} \beta_{1}^{(1)} \\
& \gamma_{5}^{\zeta_{4}}=-\frac{3}{8} \gamma_{1}^{(1)} \beta_{4}^{\zeta_{3}}-\gamma_{2}^{(1)} \beta_{3}^{\zeta_{3}}+\frac{3}{2} \gamma_{3}^{\zeta_{3}} \beta_{2}^{(1)}+\frac{3}{2} \gamma_{4}^{\zeta_{3}} \beta_{1}^{(1)}, \\
& \gamma_{5}^{\zeta_{6}}=-\frac{5}{8} \gamma_{1}^{(1)} \beta_{4}^{\zeta_{5}}+\frac{5}{2} \beta_{1}^{(1)} \gamma_{4}^{\zeta_{5}} \\
& \gamma_{5}^{\zeta_{4} \zeta_{3}}=0 \\
& \gamma_{6}^{\zeta_{4}}=-\frac{3}{10} \gamma_{1}^{(1)} \beta_{5}^{\zeta_{3}}-\frac{3}{4} \gamma_{2}^{(1)} \beta_{4}^{\zeta_{3}}-\frac{3}{2} \gamma_{3}^{(1)} \beta_{3}^{\zeta_{3}}+\frac{3}{2} \gamma_{3}^{\zeta_{3}} \beta_{3}^{(1)}+\frac{3}{2} \gamma_{4}^{\zeta_{3}} \beta_{2}^{(1)}+\frac{3}{2} \gamma_{5}^{\zeta_{3}} \beta_{1}^{(1)}, \\
& \gamma_{6}^{\zeta_{6}}=-\frac{1}{2} \gamma_{1}^{(1)} \beta_{5}^{\zeta_{5}}-\frac{5}{4} \gamma_{2}^{(1)} \beta_{4}^{\zeta_{5}}+\frac{5}{2} \beta_{2}^{(1)} \gamma_{4}^{\zeta_{5}} \\
& +\frac{5}{2} \beta_{1}^{(1)} \gamma_{5}^{\zeta_{5}}+\frac{3}{2}\left(\beta_{1}^{(1)}\right)^{2} \beta_{3}^{\zeta_{3}} \gamma_{1}^{(1)}-\frac{5}{2}\left(\beta_{1}^{(1)}\right)^{3} \gamma_{3}^{\zeta_{3}}, \\
& \gamma_{6}^{\zeta_{8}}=-\frac{7}{10} \gamma_{1}^{(1)} \beta_{5}^{\zeta_{7}}+\frac{7}{2} \beta_{1}^{(1)} \gamma_{5}^{\zeta_{7}} \\
& \gamma_{6}^{\zeta_{4} \zeta_{3}}=-\frac{3}{5} \gamma_{1}^{(1)} \beta_{5}^{\zeta_{3}^{2}}+3 \beta_{1}^{(1)} \gamma_{5}^{\zeta_{3}^{2}} \\
& \gamma_{6}^{\zeta_{4} \zeta_{5}}=0 \\
& \gamma_{6}^{\zeta_{6} \zeta_{3}}=0 \\
& \gamma_{7}^{\zeta_{4}}=-\frac{1}{4} \gamma_{1}^{(1)} \beta_{6}^{\zeta_{3}}-\frac{3}{5} \gamma_{2}^{(1)} \beta_{5}^{\zeta_{3}}-\frac{9}{8} \gamma_{3}^{(1)} \beta_{4}^{\zeta_{3}}+\frac{3}{2} \gamma_{3}^{\zeta_{3}} \beta_{4}^{(1)}-2 \gamma_{4}^{(1)} \beta_{3}^{\zeta_{3}} \\
& +\frac{3}{2} \gamma_{4}^{\zeta_{3}} \beta_{3}^{(1)}+\frac{3}{2} \gamma_{5}^{\zeta_{3}} \beta_{2}^{(1)}+\frac{3}{2} \gamma_{6}^{\zeta_{3}} \beta_{1}^{(1)}, \\
& \gamma_{7}^{\zeta_{6}}=-\frac{5}{12} \gamma_{1}^{(1)} \beta_{6}^{\zeta_{5}}-\gamma_{2}^{(1)} \beta_{5}^{\zeta_{5}}-\frac{15}{8} \gamma_{3}^{(1)} \beta_{4}^{\zeta_{5}}+\frac{5}{2} \beta_{3}^{(1)} \gamma_{4}^{\zeta_{5}}+\frac{5}{2} \beta_{2}^{(1)} \gamma_{5}^{\zeta_{5}} \\
& +\frac{5}{2} \beta_{1}^{(1)} \gamma_{6}^{\zeta_{5}}+\frac{5}{2} \beta_{1}^{(1)} \beta_{2}^{(1)} \beta_{3}^{\zeta_{3}} \gamma_{1}^{(1)}+\frac{5}{4}\left(\beta_{1}^{(1)}\right)^{2} \beta_{4}^{\zeta_{3}} \gamma_{1}^{(1)} \\
& +3\left(\beta_{1}^{(1)}\right)^{2} \beta_{3}^{\zeta_{3}} \gamma_{2}^{(1)}-\frac{15}{2}\left(\beta_{1}^{(1)}\right)^{2} \beta_{2}^{(1)} \gamma_{3}^{\zeta_{3}}-\frac{5}{2}\left(\beta_{1}^{(1)}\right)^{3} \gamma_{4}^{\zeta_{3}}, \\
& \gamma_{7}^{\zeta_{8}}=-\frac{7}{12} \gamma_{1}^{(1)} \beta_{6}^{\zeta_{7}}-\frac{7}{5} \gamma_{2}^{(1)} \beta_{5}^{\zeta_{7}}+\frac{7}{12}\left(\beta_{3}^{\zeta_{3}}\right)^{2} \gamma_{1}^{(1)}+\frac{7}{2} \beta_{2}^{(1)} \gamma_{5}^{\zeta_{7}} \\
& +\frac{7}{2} \beta_{1}^{(1)} \gamma_{6}^{\zeta_{7}}-\frac{7}{8} \beta_{1}^{(1)} \gamma_{1}^{(1)} \beta_{5}^{\zeta_{3}^{2}}-\frac{7}{8} \beta_{1}^{(1)} \beta_{3}^{\zeta_{3}} \gamma_{3}^{\zeta_{3}} \\
& +\frac{21}{8}\left(\beta_{1}^{(1)}\right)^{2} \gamma_{5}^{\zeta_{3}^{2}}+\frac{35}{8}\left(\beta_{1}^{(1)}\right)^{2} \gamma_{1}^{(1)} \beta_{4}^{\zeta_{5}}-\frac{35}{4}\left(\beta_{1}^{(1)}\right)^{3} \gamma_{4}^{\zeta_{5}},
\end{aligned}
$$




$$
\begin{aligned}
\gamma_{7}^{\zeta_{10}} & =-\frac{3}{4} \gamma_{1}^{(1)} \beta_{6}^{\zeta_{9}}+\frac{9}{2} \beta_{1}^{(1)} \gamma_{6}^{\zeta_{9}} \\
\gamma_{7}^{\zeta_{4} \zeta_{3}} & =-\frac{1}{2} \gamma_{1}^{(1)} \beta_{6}^{\zeta_{3}^{2}}-\frac{6}{5} \gamma_{2}^{(1)} \beta_{5}^{\zeta_{3}^{2}}+\frac{3}{8} \gamma_{3}^{\zeta_{3}} \beta_{4}^{\zeta_{3}}-\frac{1}{2} \gamma_{4}^{\zeta_{3}} \beta_{3}^{\zeta_{3}}+3 \beta_{2}^{(1)} \gamma_{5}^{\zeta_{3}^{2}}+3 \beta_{1}^{(1)} \gamma_{6}^{\zeta_{3}^{2}} \\
\gamma_{7}^{\zeta_{4} \zeta_{5}} & =\frac{5}{4} \gamma_{1}^{(1)} \beta_{6}^{\bar{\zeta}_{5,3}}-\frac{1}{4} \gamma_{1}^{(1)} \beta_{6}^{\zeta_{3} \zeta_{5}}+\frac{3}{2} \gamma_{3}^{\zeta_{3}} \beta_{4}^{\zeta_{5}}-2 \beta_{3}^{\zeta_{3}} \gamma_{4}^{\zeta_{5}}-\frac{15}{2} \beta_{1}^{(1)} \gamma_{6}^{\zeta_{5,3}}+\frac{3}{2} \beta_{1}^{(1)} \gamma_{6}^{\zeta_{3} \zeta_{5}} \\
\gamma_{7}^{\zeta_{4} \zeta_{7}} & =0 \\
\gamma_{7}^{\zeta_{6} \zeta_{3}} & =-\frac{5}{12} \gamma_{1}^{(1)} \beta_{6}^{\zeta_{3} \zeta_{5}}-\frac{15}{8} \gamma_{3}^{\zeta_{3}} \beta_{4}^{\zeta_{5}}+\frac{5}{2} \beta_{3}^{\zeta_{3}} \gamma_{4}^{\zeta_{5}}+\frac{5}{2} \beta_{1}^{(1)} \gamma_{6}^{\zeta_{3} \zeta_{5}} \\
\gamma_{7}^{\zeta_{6} \zeta_{5}} & =0 \\
\gamma_{7}^{\zeta_{8} \zeta_{3}} & =0 \\
\gamma_{7}^{\zeta_{4} \zeta_{3}^{2}} & =-\frac{3}{4} \gamma_{1}^{(1)} \beta_{6}^{\zeta_{3}^{3}}+\frac{9}{2} \beta_{1}^{(1)} \gamma_{6}^{\zeta_{3}^{3}}
\end{aligned}
$$

The results for $\pi$-dependent contributions to a $\beta$-function are obtained from the above relations by a formal replacement of $\gamma$ by $\beta$ in every term. For instance, the 6 and 7 -loop $\pi$-dependent contributions read:

$$
\begin{aligned}
\beta_{6}^{\zeta_{4}} & =\frac{6}{5} \beta_{1}^{(1)} \beta_{5}^{\zeta_{3}}+\frac{3}{4} \beta_{2}^{(1)} \beta_{4}^{\zeta_{3}} \\
\beta_{6}^{\zeta_{6}} & =2 \beta_{1}^{(1)} \beta_{5}^{\zeta_{5}}-\left(\beta_{1}^{(1)}\right)^{3} \beta_{3}^{\zeta_{3}}+\frac{5}{4} \beta_{2}^{(1)} \beta_{4}^{\zeta_{5}} \\
\beta_{6}^{\zeta_{8}} & =\frac{14}{5} \beta_{1}^{(1)} \beta_{5}^{\zeta_{7}} \\
\beta_{6}^{\zeta_{4} \zeta_{3}} & =\frac{12}{5} \beta_{1}^{(1)} \beta_{5}^{\zeta_{3}^{2}} \\
\beta_{6}^{\zeta_{4} \zeta_{5}} & =0 \\
\beta_{6}^{\zeta_{6} \zeta_{3}} & =0 \\
\beta_{7}^{\zeta_{4}} & =\frac{5}{4} \beta_{1}^{(1)} \beta_{6}^{\zeta_{3}}+\frac{9}{10} \beta_{2}^{(1)} \beta_{5}^{\zeta_{3}}+\frac{3}{8} \beta_{3}^{(1)} \beta_{4}^{\zeta_{3}}-\frac{1}{2} \beta_{3}^{\zeta_{3}} \beta_{4}^{(1)}, \\
\beta_{7}^{\zeta_{6}} & =\frac{25}{12} \beta_{1}^{(1)} \beta_{6}^{\zeta_{5}}+\frac{3}{2} \beta_{2}^{(1)} \beta_{5}^{\zeta_{5}}-\frac{5}{4} \beta_{4}^{\zeta_{3}}\left(\beta_{1}^{(1)}\right)^{3}+\frac{5}{8} \beta_{3}^{(1)} \beta_{4}^{\zeta_{5}}-2 \beta_{3}^{\zeta_{3}} \beta_{2}^{(1)}\left(\beta_{1}^{(1)}\right)^{2}, \\
\beta_{7}^{\zeta_{8}} & =\frac{35}{12} \beta_{1}^{(1)} \beta_{6}^{\zeta_{7}}+\frac{7}{4}\left(\beta_{1}^{(1)}\right)^{2} \beta_{5}^{\zeta_{3}^{2}}-\frac{35}{8}\left(\beta_{1}^{(1)}\right)^{3} \beta_{4}^{\zeta_{5}}+\frac{21}{10} \beta_{2}^{(1)} \beta_{5}^{\zeta_{7}}-\frac{7}{24}\left(\beta_{3}^{\zeta_{3}}\right)^{2} \beta_{1}^{(1)}, \\
\beta_{7}^{\zeta_{10}} & =\frac{15}{4} \beta_{1}^{(1)} \beta_{6}^{\zeta_{9}}, \\
\beta_{7}^{\zeta_{4} \zeta_{3}} & =\frac{5}{2} \beta_{1}^{(1)} \beta_{6}^{\zeta_{3}^{2}}+\frac{9}{5} \beta_{2}^{(1)} \beta_{5}^{\zeta_{3}^{2}}-\frac{1}{8} \beta_{3}^{\zeta_{3}} \beta_{4}^{\zeta_{3}}, \\
\beta_{7}^{\zeta_{4} \zeta_{5}} & =-\frac{25}{4} \beta_{1}^{(1)} \beta_{6}^{\zeta_{5,3}}+\frac{5}{4} \beta_{1}^{(1)} \beta_{6}^{\zeta_{3} \zeta_{5}}-\frac{1}{2} \beta_{3}^{\zeta_{3}} \beta_{4}^{\zeta_{5}} \\
\beta_{7}^{\zeta_{4} \zeta_{7}} & =0 \\
\beta_{7}^{\zeta_{6} \zeta_{3}} & =\frac{25}{12} \beta_{1}^{(1)} \beta_{6}^{\zeta_{3} \zeta_{5}}+\frac{5}{8} \beta_{3}^{\zeta_{3}} \beta_{4}^{\zeta_{5}}, \\
\beta_{7}^{\zeta_{6} \zeta_{5}} & =0 \\
& 0
\end{aligned}
$$




$$
\begin{aligned}
& \beta_{7}^{\zeta_{8} \zeta_{3}}=0, \\
& \beta_{7}^{\zeta_{4} \zeta_{3}^{2}}=\frac{15}{4} \beta_{1}^{(1)} \beta_{6}^{\zeta_{3}^{3}} .
\end{aligned}
$$

\subsection{Tests at 7 loops}

With eqs. (4.1)-(4.38) we have been able to reproduce successfully all $\pi$-dependent constants appearing in the $\beta$-function and anomalous dimensions $\gamma_{m}$ and $\gamma_{2}$ of the $O(n) \varphi^{4}$ model which all are known at 7 loops from [2]. In addition, we have checked that the $\pi$ dependent contributions to the terms of order $n_{f}^{6} \alpha_{s}^{7}$ in the QCD $\beta$-function as well as to the terms of order $n_{f}^{6} \alpha_{s}^{7}$ and of order $n_{f}^{5} \alpha_{s}^{7}$ contributing to the quark mass $\mathrm{AD}$ (all computed in [30-32]) within the framework of large $N_{f}[3,33-36]$ approach are in full agreement with the constraints listed above.

Numerous successful tests at 4,5 and 6 loops have been presented in [1].

\section{Hatted representation for 7 -loop p-integrals and the $\pi^{12}$ subtlety}

Motivated by the success of our derivation of the hatted representation for the 6-loop case we have decided to look on the next, 7-loop level. Within our approach this requires the knowledge of the $\epsilon$-expansion of the 4-loop master integrals presented in [18] up to the transcendental weight 13 . In principle, the methods employed by Lee and A. and V. Smirnovs are powerful enough to find such an expansion. One of the authors of [18] has provided us with $\epsilon$-expansions for all 4-loop master p-integrals up to and including weight 13 .

In fact, we have (well, almost) succeeded in constructing the hatted representation for the subset $\mathcal{P}_{4} / \epsilon^{3}$ of $\mathcal{P}_{7}$. Our results are presented below. ${ }^{9}$

$$
\begin{aligned}
& \underset{L=7}{\hat{\zeta}_{3}}:=\underset{L=6}{\hat{\zeta}_{3}}+\frac{1705 \epsilon^{9}}{2} \zeta_{12}, \\
& \hat{\zeta}_{L=7}:=\underset{L=6}{\hat{\zeta}_{5}}-\frac{2805 \epsilon^{7}}{4} \zeta_{12}, \\
& \frac{\hat{\zeta}_{7}}{L=7}:=\frac{\hat{\zeta}_{7}}{L=6}+231 \epsilon^{5} \zeta_{12}, \\
& \hat{\zeta}_{5=7}:=\frac{\hat{\zeta}_{5,3}}{L=6}+? \zeta_{12}-\frac{105 \epsilon^{5}}{2} \zeta_{5} \zeta_{8}, \\
& \hat{\zeta}_{L=7}^{\hat{\zeta}_{9}}:=\underset{L=6}{\hat{\zeta}_{9}}-\frac{165 \epsilon^{3}}{4} \zeta_{12} \\
& \underbrace{\hat{\zeta}_{7,3}}_{L=7}:=\underbrace{\zeta_{7,3}}_{L=6}-? \zeta_{12}+\epsilon^{3}\left(\frac{105}{2} \zeta_{5} \zeta_{8}+35 \zeta_{6} \zeta_{7}\right) \text {, }
\end{aligned}
$$

\footnotetext{
${ }^{9}$ Note that the hatted representation of single odd zetas displayed in eqs. ((5.1), (5.2), (5.3), (5.5), (5.7) and (5.10) is in agreement with the recent findings of [37].
} 


$$
\begin{aligned}
& \hat{\zeta}_{L=7}:=\hat{\zeta}_{L=6}^{\hat{\zeta}_{11}}+\frac{11 \epsilon}{2} \zeta_{12} \\
& \hat{\zeta}_{5=7,3,3}:=\frac{\hat{\zeta}_{5,3,3}}{L=6}+? \zeta_{12}+\frac{3 \epsilon}{2} \zeta_{4} \zeta_{5,3}-\frac{105 \epsilon^{2}}{16} \zeta_{5} \zeta_{8}, \\
& \frac{\hat{\zeta}_{9,3}}{L=7}:=\zeta_{9,3}-\frac{75 \epsilon}{2} \zeta_{6} \zeta_{7}-21 \epsilon \zeta_{5} \zeta_{8}-\frac{81 \epsilon}{2} \zeta_{4} \zeta_{9}, \\
& \frac{\hat{\zeta}_{13}}{L=7}:=\zeta_{13}, \\
& \hat{\zeta}_{5=7}^{\hat{\zeta}_{5,3}}:=\zeta_{5,5,3}+\frac{145}{12} \zeta_{5} \zeta_{8}+25 \zeta_{4} \zeta_{9}+\frac{275}{2} \zeta_{2} \zeta_{11}, \\
& \frac{\hat{\zeta}_{7,3,3}}{L=7}:=\zeta_{7,3,3}-4 \zeta_{6} \zeta_{7}+\frac{29}{2} \zeta_{5} \zeta_{8}+28 \zeta_{4} \zeta_{9}+\frac{407}{2} \zeta_{2} \zeta_{11}, \\
& \frac{\hat{\zeta}_{6,4,1,1}}{L=7}:=\zeta_{6,4,1,1}+? \zeta_{12}-\frac{3}{2} \zeta_{4} \zeta_{5,3}+\frac{1}{2} \zeta_{3} \zeta_{4} \zeta_{5}+\frac{9}{4} \zeta_{3}^{2} \zeta_{6}-3 \zeta_{2} \zeta_{7,3}-\frac{7}{2} \zeta_{2} \zeta_{5}^{2}-10 \zeta_{2} \zeta_{3} \zeta_{7} \\
& +\epsilon\left(\frac{5665}{32} \zeta_{6} \zeta_{7}+\frac{203}{2} \zeta_{5} \zeta_{8}+\frac{1799}{12} \zeta_{4} \zeta_{9}-\frac{799}{16} \zeta_{3} \zeta_{10}+\frac{1}{2} \zeta_{3}^{3} \zeta_{4}\right) .
\end{aligned}
$$

The meaning of the question mark in front of $\zeta_{12}$ in eqs. (5.4), (5.8) and (5.13) for hatted form of multiple zeta objects is as follows. Every integral from the set $\mathcal{P}_{4} / \epsilon^{3}$ can either include at least one (or more) multiple zeta values from the collection $\bar{\zeta}_{5,3}, \bar{\zeta}_{7,3}, \bar{\zeta}_{5,3,3}, \bar{\zeta}_{7,3,3}$ and $\bar{\zeta}_{6,4,1,1}$ or not. Thus, the whole set $\mathcal{P}_{4} / \epsilon^{3}$ can be represented as a union of two (nonintersecting!) subsets, namely, a simple one, $\mathcal{S}_{4} / \epsilon^{3}$, (that is without any dependence on multiple zeta values) and the rest $\mathcal{N}_{4} / \epsilon^{3}$.

The fact is that the hatted representation does exists for all p-integrals $\mathcal{S}_{4} / \epsilon^{3}$, while there is no way to replace the question marks in eqs. above by some coefficients in order to meet eq. (2.5) for the p-integrals from $\mathcal{N}_{4} / \epsilon^{3}$. On the other hand, if we formally set to zero all terms proportional to $\zeta_{12}$ in eqs. (5.1)-(5.13), then eq. (2.5) will be valid for the whole set $\mathcal{P}_{4} / \epsilon^{3}$ "modulo" terms proportional to $\zeta_{12}$.

It is quite remarkable that the distinguished role of $\zeta_{12}$ has been already established in [2] as a result of direct analytical calculations of quite complicated convergent 7-loop p-integrals.

Thus, we observe a nontrivial interplay between higher terms in the $\epsilon$-expansion of 4-loop p-integrals and 7-loop finite p-integrals.

Certainly, the subset of the 7-loop p-integrals which has led to eqs. (5.1)-(5.13) is rather limited and our conclusions about $\pi$-structure of $\mathcal{P}_{7}$ are not final. In principle, we can outline 3 possible scenarios.

Scenario 1 (pessimistic). There is no hatted representation for the set $\mathcal{P}_{7}$.

Scenario 2 (conservative). The master p-integrals from the difference $\mathcal{P}_{7} \backslash \mathcal{P}_{4} / \epsilon^{3}$ can be presented in the hatted form modulo (explicitly) $\pi$-proportional terms with weight more or equal 12 . 
Scenario 3 (optimistic). The master p-integrals from the difference $\mathcal{P}_{7} \backslash \mathcal{P}_{4} / \epsilon^{3}$ can be presented in the hatted form modulo $\zeta_{12}$.

\section{$6 \pi$-dependence of 8-loop $\beta$-functions and AD}

In this section we assume the conservative Scenario 2 and extend (following generic prescriptions elaborated in [1]) the predictions from section 4 by one more loop for $\pi$-dependent terms with the transcendental weight not exceeding 11 . The results read:

$$
\begin{aligned}
& \gamma_{8}^{\zeta_{4}}=-\frac{3}{14} \gamma_{1}^{(1)} \beta_{7}^{\zeta_{3}}-\frac{1}{2} \gamma_{2}^{(1)} \beta_{6}^{\zeta_{3}}-\frac{9}{10} \gamma_{3}^{(1)} \beta_{5}^{\zeta_{3}}+\frac{3}{2} \gamma_{3}^{\zeta_{3}} \beta_{5}^{(1)}-\frac{3}{2} \gamma_{4}^{(1)} \beta_{4}^{\zeta_{3}} \\
& +\frac{3}{2} \gamma_{4}^{\zeta_{3}} \beta_{4}^{(1)}-\frac{5}{2} \gamma_{5}^{(1)} \beta_{3}^{\zeta_{3}}+\frac{3}{2} \gamma_{5}^{\zeta_{3}} \beta_{3}^{(1)}+\frac{3}{2} \gamma_{6}^{\zeta_{3}} \beta_{2}^{(1)}+\frac{3}{2} \gamma_{7}^{\zeta_{3}} \beta_{1}^{(1)} \\
& \gamma_{8}^{\zeta_{6}}=-\frac{5}{14} \gamma_{1}^{(1)} \beta_{7}^{\zeta_{5}}-\frac{5}{6} \gamma_{2}^{(1)} \beta_{6}^{\zeta_{5}}-\frac{3}{2} \gamma_{3}^{(1)} \beta_{5}^{\zeta_{5}}-\frac{5}{2} \gamma_{4}^{(1)} \beta_{4}^{\zeta_{5}}+\frac{5}{2} \beta_{4}^{(1)} \gamma_{4}^{\zeta_{5}}+\frac{5}{2} \beta_{3}^{(1)} \gamma_{5}^{\zeta_{5}} \\
& +\frac{5}{2} \beta_{2}^{(1)} \gamma_{6}^{\zeta_{5}}+\frac{15}{14}\left(\beta_{2}^{(1)}\right)^{2} \beta_{3}^{\zeta_{3}} \gamma_{1}^{(1)}+\frac{5}{2} \beta_{1}^{(1)} \gamma_{7}^{\zeta_{5}} \\
& +\frac{15}{7} \beta_{1}^{(1)} \beta_{3}^{(1)} \beta_{3}^{\zeta_{3}} \gamma_{1}^{(1)}+\frac{15}{7} \beta_{1}^{(1)} \beta_{2}^{(1)} \beta_{4}^{\zeta_{3}} \gamma_{1}^{(1)}+5 \beta_{1}^{(1)} \beta_{2}^{(1)} \beta_{3}^{\zeta_{3}} \gamma_{2}^{(1)}-\frac{15}{2} \beta_{1}^{(1)}\left(\beta_{2}^{(1)}\right)^{2} \gamma_{3}^{\zeta_{3}} \\
& +\frac{15}{14}\left(\beta_{1}^{(1)}\right)^{2} \beta_{5}^{\zeta_{3}} \gamma_{1}^{(1)}+\frac{5}{2}\left(\beta_{1}^{(1)}\right)^{2} \beta_{4}^{\zeta_{3}} \gamma_{2}^{(1)}+\frac{9}{2}\left(\beta_{1}^{(1)}\right)^{2} \beta_{3}^{\zeta_{3}} \gamma_{3}^{(1)}-\frac{15}{2}\left(\beta_{1}^{(1)}\right)^{2} \beta_{3}^{(1)} \gamma_{3}^{\zeta_{3}} \\
& -\frac{15}{2}\left(\beta_{1}^{(1)}\right)^{2} \beta_{2}^{(1)} \gamma_{4}^{\zeta_{3}}-\frac{5}{2}\left(\beta_{1}^{(1)}\right)^{3} \gamma_{5}^{\zeta_{3}} \\
& \gamma_{8}^{\zeta_{8}}=-\frac{1}{2} \gamma_{1}^{(1)} \beta_{7}^{\zeta_{7}}-\frac{7}{6} \gamma_{2}^{(1)} \beta_{6}^{\zeta_{7}}-\frac{21}{10} \gamma_{3}^{(1)} \beta_{5}^{\zeta_{7}}+\beta_{3}^{\zeta_{3}} \beta_{4}^{\zeta_{3}} \gamma_{1}^{(1)}+\frac{35}{24}\left(\beta_{3}^{\zeta_{3}}\right)^{2} \gamma_{2}^{(1)} \\
& +\frac{7}{2} \beta_{3}^{(1)} \gamma_{5}^{\zeta_{7}}+\frac{7}{2} \beta_{2}^{(1)} \gamma_{6}^{\zeta_{7}}-\frac{3}{4} \beta_{2}^{(1)} \gamma_{1}^{(1)} \beta_{5}^{\zeta_{3}^{2}}-\frac{7}{4} \beta_{2}^{(1)} \beta_{3}^{\zeta_{3}} \gamma_{3}^{\zeta_{3}} \\
& +\frac{7}{2} \beta_{1}^{(1)} \gamma_{7}^{\zeta_{7}}-\frac{3}{4} \beta_{1}^{(1)} \gamma_{1}^{(1)} \beta_{6}^{\zeta_{3}^{2}}-\frac{7}{4} \beta_{1}^{(1)} \gamma_{2}^{(1)} \beta_{5}^{\zeta_{3}^{2}}-\frac{7}{4} \beta_{1}^{(1)} \beta_{3}^{\zeta_{3}} \gamma_{4}^{\zeta_{3}} \\
& +\frac{21}{4} \beta_{1}^{(1)} \beta_{2}^{(1)} \gamma_{5}^{\zeta_{3}^{2}}+\frac{15}{2} \beta_{1}^{(1)} \beta_{2}^{(1)} \gamma_{1}^{(1)} \beta_{4}^{\zeta_{5}}+\frac{21}{8}\left(\beta_{1}^{(1)}\right)^{2} \gamma_{6}^{\zeta_{3}^{2}}+\frac{15}{4}\left(\beta_{1}^{(1)}\right)^{2} \gamma_{1}^{(1)} \beta_{5}^{\zeta_{5}} \\
& +\frac{35}{4}\left(\beta_{1}^{(1)}\right)^{2} \gamma_{2}^{(1)} \beta_{4}^{\zeta_{5}}-\frac{105}{4}\left(\beta_{1}^{(1)}\right)^{2} \beta_{2}^{(1)} \gamma_{4}^{\zeta_{5}}-\frac{35}{4}\left(\beta_{1}^{(1)}\right)^{3} \gamma_{5}^{\zeta_{5}} \\
& -\frac{15}{2}\left(\beta_{1}^{(1)}\right)^{4} \beta_{3}^{\zeta_{3}} \gamma_{1}^{(1)}+\frac{21}{2}\left(\beta_{1}^{(1)}\right)^{5} \gamma_{3}^{\zeta_{3}} \\
& \gamma_{8}^{\zeta_{10}}=-\frac{9}{14} \gamma_{1}^{(1)} \beta_{7}^{\zeta_{9}}-\frac{3}{2} \gamma_{2}^{(1)} \beta_{6}^{\zeta_{9}}+\frac{11}{7} \beta_{3}^{\zeta_{3}} \gamma_{1}^{(1)} \beta_{4}^{\zeta_{5}}+\frac{9}{2} \beta_{2}^{(1)} \gamma_{6}^{\zeta_{9}}+\frac{9}{2} \beta_{1}^{(1)} \gamma_{7}^{\zeta_{9}}+\frac{415}{188} \beta_{1}^{(1)} \gamma_{1}^{(1)} \beta_{6}^{\bar{\zeta}_{5,3}} \\
& -\frac{33}{28} \beta_{1}^{(1)} \gamma_{1}^{(1)} \beta_{6}^{\zeta_{3} \zeta_{5}}-\frac{11}{4} \beta_{1}^{(1)} \beta_{3}^{\zeta_{3}} \gamma_{4}^{\zeta_{5}}-\frac{2905}{376}\left(\beta_{1}^{(1)}\right)^{2} \gamma_{6}^{\bar{\zeta}_{5,3}} \\
& +\frac{33}{8}\left(\beta_{1}^{(1)}\right)^{2} \gamma_{6}^{\zeta_{3} \zeta_{5}}+9\left(\beta_{1}^{(1)}\right)^{2} \gamma_{1}^{(1)} \beta_{5}^{\zeta_{7}}-21\left(\beta_{1}^{(1)}\right)^{3} \gamma_{5}^{\zeta_{7}}, \\
& \gamma_{8}^{\zeta_{4} \zeta_{3}}=-\frac{3}{7} \gamma_{1}^{(1)} \beta_{7}^{\zeta_{3}^{2}}-\gamma_{2}^{(1)} \beta_{6}^{\zeta_{3}^{2}}-\frac{9}{5} \gamma_{3}^{(1)} \beta_{5}^{\zeta_{3}^{2}}+\frac{3}{5} \gamma_{3}^{\zeta_{3}} \beta_{5}^{\zeta_{3}}+3 \beta_{3}^{(1)} \gamma_{5}^{\zeta_{3}^{2}} \\
& -\gamma_{5}^{\zeta_{3}} \beta_{3}^{\zeta_{3}}+3 \beta_{2}^{(1)} \gamma_{6}^{\zeta_{3}^{2}}+3 \beta_{1}^{(1)} \gamma_{7}^{\zeta_{3}^{2}}
\end{aligned}
$$




$$
\begin{aligned}
& \gamma_{8}^{\zeta_{4} \zeta_{5}}=\frac{15}{14} \gamma_{1}^{(1)} \beta_{7}^{\bar{\zeta}_{5,3}}-\frac{3}{14} \gamma_{1}^{(1)} \beta_{7}^{\zeta_{3} \zeta_{5}}+\frac{5}{2} \gamma_{2}^{(1)} \beta_{6}^{\bar{\zeta}_{5,3}}-\frac{1}{2} \gamma_{2}^{(1)} \beta_{6}^{\zeta_{3} \zeta_{5}}+\frac{3}{2} \gamma_{3}^{\zeta_{3}} \beta_{5}^{\zeta_{5}} \\
& -\frac{3}{2} \beta_{4}^{\zeta_{3}} \gamma_{4}^{\zeta_{5}}+\frac{3}{2} \gamma_{4}^{\zeta_{3}} \beta_{4}^{\zeta_{5}}-\frac{5}{2} \beta_{3}^{\zeta_{3}} \gamma_{5}^{\zeta_{5}}-\frac{15}{2} \beta_{2}^{(1)} \bar{\gamma}_{6}^{\zeta_{5,3}} \\
& +\frac{3}{2} \beta_{2}^{(1)} \gamma_{6}^{\zeta_{3} \zeta_{5}}-\frac{15}{2} \beta_{1}^{(1)} \bar{\zeta}_{7} \overline{5}_{5,3}+\frac{3}{2} \beta_{1}^{(1)} \gamma_{7}^{\zeta_{3} \zeta_{5}} \\
& \gamma_{8}^{\zeta_{4} \zeta_{7}}=3 \gamma_{1}^{(1)} \beta_{7}^{\bar{\zeta}_{7,3}}-\frac{3}{14} \gamma_{1}^{(1)} \beta_{7}^{\zeta_{3} \zeta_{7}}+\frac{3}{2} \gamma_{3}^{\zeta_{3}} \beta_{5}^{\zeta_{7}}-\frac{5}{2} \beta_{3}^{\zeta_{3}} \gamma_{5}^{\zeta_{7}}-21 \beta_{1}^{(1)} \gamma_{7}^{\bar{\zeta}_{7,3}}+\frac{3}{2} \beta_{1}^{(1)} \gamma_{7}^{\zeta_{3} \zeta_{7}}, \\
& \gamma_{8}^{\zeta_{6} \zeta_{3}}=-\frac{5}{14} \gamma_{1}^{(1)} \beta_{7}^{\zeta_{3} \zeta_{5}}-\frac{5}{6} \gamma_{2}^{(1)} \beta_{6}^{\zeta_{3} \zeta_{5}}-\frac{3}{2} \gamma_{3}^{\zeta_{3}} \beta_{5}^{\zeta_{5}}+\frac{5}{2} \beta_{4}^{\zeta_{3}} \gamma_{4}^{\zeta_{5}}-\frac{5}{2} \gamma_{4}^{\zeta_{3}} \beta_{4}^{\zeta_{5}} \\
& +\frac{5}{2} \beta_{3}^{\zeta_{3}} \gamma_{5}^{\zeta_{5}}+\frac{5}{2} \beta_{2}^{(1)} \gamma_{6}^{\zeta_{3} \zeta_{5}}+\frac{5}{2} \beta_{1}^{(1)} \gamma_{7}^{\zeta_{3} \zeta_{5}}+\frac{15}{7} \beta_{1}^{(1)}\left(\beta_{3}^{\zeta_{3}}\right)^{2} \gamma_{1}^{(1)} \\
& +\frac{15}{7}\left(\beta_{1}^{(1)}\right)^{2} \gamma_{1}^{(1)} \beta_{5}^{\zeta_{3}^{2}}-3\left(\beta_{1}^{(1)}\right)^{2} \beta_{3}^{\zeta_{3}} \gamma_{3}^{\zeta_{3}}-5\left(\beta_{1}^{(1)}\right)^{3} \gamma_{5}^{\zeta_{3}^{2}}, \\
& \gamma_{8}^{\zeta_{6} \zeta_{5}}=\frac{15}{7} \gamma_{1}^{(1)} \beta_{7}^{\bar{\zeta}_{7,3}}-\frac{5}{7} \gamma_{1}^{(1)} \beta_{7}^{\zeta_{5}^{2}}-15 \beta_{1}^{(1)} \gamma_{7}^{\bar{\zeta}_{7,3}}+5 \beta_{1}^{(1)} \gamma_{7}^{\zeta_{5}^{2}} \\
& \gamma_{8}^{\zeta_{8} \zeta_{3}}=-\frac{1}{2} \gamma_{1}^{(1)} \beta_{7}^{\zeta_{3} \zeta_{7}}-\frac{21}{10} \gamma_{3}^{\zeta_{3}} \beta_{5}^{\zeta_{7}}+\frac{7}{2} \beta_{3}^{\zeta_{3}} \gamma_{5}^{\zeta_{7}}+\frac{7}{2} \beta_{1}^{(1)} \gamma_{7}^{\zeta_{3} \zeta_{7}}-\frac{9}{4} \beta_{1}^{(1)} \gamma_{1}^{(1)} \beta_{6}^{\zeta_{3}^{3}}+\frac{63}{8}\left(\beta_{1}^{(1)}\right)^{2} \gamma_{6}^{\zeta_{3}^{3}} \\
& \beta_{8}^{\zeta_{4}}=\frac{9}{7} \beta_{1}^{(1)} \beta_{7}^{\zeta_{3}}+\beta_{2}^{(1)} \beta_{6}^{\zeta_{3}}+\frac{3}{5} \beta_{3}^{(1)} \beta_{5}^{\zeta_{3}}-\beta_{3}^{\zeta_{3}} \beta_{5}^{(1)}, \\
& \beta_{8}^{\zeta_{6}}=\frac{15}{7} \beta_{1}^{(1)} \beta_{7}^{\zeta_{5}}+\frac{5}{3} \beta_{2}^{(1)} \beta_{6}^{\zeta_{5}}-\frac{20}{7} \beta_{2}^{(1)}\left(\beta_{1}^{(1)}\right)^{2} \beta_{4}^{\zeta_{3}}-\frac{10}{7} \beta_{5}^{\zeta_{3}}\left(\beta_{1}^{(1)}\right)^{3} \\
& +\beta_{3}^{(1)} \beta_{5}^{\zeta_{5}}-\frac{10}{7} \beta_{3}^{\zeta_{3}}\left(\beta_{2}^{(1)}\right)^{2} \beta_{1}^{(1)}-\frac{6}{7} \beta_{3}^{\zeta_{3}} \beta_{3}^{(1)}\left(\beta_{1}^{(1)}\right)^{2}, \\
& \beta_{8}^{\zeta_{8}}=3 \beta_{1}^{(1)} \beta_{7}^{\zeta_{7}}+\frac{15}{8}\left(\beta_{1}^{(1)}\right)^{2} \beta_{6}^{\zeta_{3}^{2}}-5\left(\beta_{1}^{(1)}\right)^{3} \beta_{5}^{\zeta_{5}}+\frac{7}{3} \beta_{2}^{(1)} \beta_{6}^{\zeta_{7}}+\frac{11}{4} \beta_{2}^{(1)} \beta_{1}^{(1)} \beta_{5}^{\zeta_{3}^{2}} \\
& -10 \beta_{2}^{(1)}\left(\beta_{1}^{(1)}\right)^{2} \beta_{4}^{\zeta_{5}}+\frac{7}{5} \beta_{3}^{(1)} \beta_{5}^{\zeta_{7}}-\frac{3}{4} \beta_{3}^{\zeta_{3}} \beta_{1}^{(1)} \beta_{4}^{\zeta_{3}}+3 \beta_{3}^{\zeta_{3}}\left(\beta_{1}^{(1)}\right)^{5}-\frac{7}{24}\left(\beta_{3}^{\zeta_{3}}\right)^{2} \beta_{2}^{(1)}, \\
& \beta_{8}^{\zeta_{10}}=\frac{27}{7} \beta_{1}^{(1)} \beta_{7}^{\zeta_{9}}-\frac{2075}{376}\left(\beta_{1}^{(1)}\right)^{2} \beta_{6}^{\bar{\zeta}_{5,3}}+\frac{165}{56}\left(\beta_{1}^{(1)}\right)^{2} \beta_{6}^{\zeta_{3} \zeta_{5}} \\
& -12\left(\beta_{1}^{(1)}\right)^{3} \beta_{5}^{\zeta_{7}}+3 \beta_{2}^{(1)} \beta_{6}^{\zeta_{9}}-\frac{33}{28} \beta_{3}^{\zeta_{3}} \beta_{1}^{(1)} \beta_{4}^{\zeta_{5}}, \\
& \beta_{8}^{\zeta_{4} \zeta_{3}}=\frac{18}{7} \beta_{1}^{(1)} \beta_{7}^{\zeta_{3}^{2}}+2 \beta_{2}^{(1)} \beta_{6}^{\zeta_{3}^{2}}+\frac{6}{5} \beta_{3}^{(1)} \beta_{5}^{\zeta_{3}^{2}}-\frac{2}{5} \beta_{3}^{\zeta_{3}} \beta_{5}^{\zeta_{3}}, \\
& \beta_{8}^{\zeta_{4} \zeta_{5}}=-\frac{45}{7} \beta_{1}^{(1)} \beta_{7}^{\bar{\zeta}_{5,3}}+\frac{9}{7} \beta_{1}^{(1)} \beta_{7}^{\zeta_{3} \zeta_{5}}-5 \beta_{2}^{(1)} \beta_{6}^{\bar{\zeta}_{5,3}}+\beta_{2}^{(1)} \beta_{6}^{\zeta_{3} \zeta_{5}}-\beta_{3}^{\zeta_{3}} \beta_{5}^{\zeta_{5}}, \\
& \beta_{8}^{\zeta_{4} \zeta_{7}}=-18 \beta_{1}^{(1)} \beta_{7}^{\bar{\zeta}_{7,3}}+\frac{9}{7} \beta_{1}^{(1)} \beta_{7}^{\zeta_{3} \zeta_{7}}-\beta_{3}^{\zeta_{3}} \beta_{5}^{\zeta_{7}}, \\
& \beta_{8}^{\zeta_{6} \zeta_{3}}=\frac{15}{7} \beta_{1}^{(1)} \beta_{7}^{\zeta_{3} \zeta_{5}}-\frac{20}{7}\left(\beta_{1}^{(1)}\right)^{3} \beta_{5}^{\zeta_{3}^{2}}+\frac{5}{3} \beta_{2}^{(1)} \beta_{6}^{\zeta_{3} \zeta_{5}}+\beta_{3}^{\zeta_{3}} \beta_{5}^{\zeta_{5}}-\frac{6}{7}\left(\beta_{3}^{\zeta_{3}}\right)^{2}\left(\beta_{1}^{(1)}\right)^{2}, \\
& \beta_{8}^{\zeta_{6} \zeta_{5}}=-\frac{90}{7} \beta_{1}^{(1)} \beta_{7}^{\bar{\zeta}_{7,3}}+\frac{30}{7} \beta_{1}^{(1)} \beta_{7}^{\zeta_{5}^{2}}, \\
& \beta_{8}^{\zeta_{8} \zeta_{3}}=3 \beta_{1}^{(1)} \beta_{7}^{\zeta_{3} \zeta_{7}}+\frac{45}{8}\left(\beta_{1}^{(1)}\right)^{2} \beta_{6}^{\zeta_{3}^{3}}+\frac{7}{5} \beta_{3}^{\zeta_{3}} \beta_{5}^{\zeta_{7}}, \\
& \beta_{8}^{\zeta_{4} \zeta_{3}^{2}}=\frac{27}{7} \beta_{1}^{(1)} \beta_{7}^{\zeta_{3}^{3}}+3 \beta_{2}^{(1)} \beta_{6}^{\zeta_{3}^{3}}+\frac{1}{5} \beta_{3}^{\zeta_{3}} \beta_{5}^{\zeta_{3}^{2}} .
\end{aligned}
$$




\subsection{Tests at 8 loops}

Here we summarize all currently available evidence supporting assumptions (that is scenarios 2 and 3) leading to eqs. (6.1)-(6.21).

First of all, we have checked that currently known 8-loop results for ADs and $\beta$ functions are in full agreement to our predictions. Namely, we have successfully checked the following cases.

- Contributions of order $\alpha_{s}^{8} N_{f}^{7}$ to the QCD $\beta$-function [30].

- Contributions of orders $\alpha_{s}^{8} N_{f}^{7}$ and $\alpha_{s}^{8} N_{f}^{6}$ to the QCD quark mass anomalous dimension [31, 32].

- Contributions of order $g^{8} n^{7}$ and $g^{8} n^{6}$ to the $\beta$-function, the field anomalous dimension and to the mass anomalous dimension of the scalar $O(n) \phi^{4}$ theory [36].

The above list give some support to the conservative Scenario 2. In fact, there is an argument in favor of even less conservative optimistic Scenario 3. Indeed, according our definitions the value of any convergent (and expressible in terms of multiple zeta values only) 7-loop p-integral at $D=4$ should become completely $\pi$-free (modulo terms proportional to $\pi^{12}$ ) if rewritten in terms of the generators

$$
\zeta_{3}, \zeta_{5}, \zeta_{7}, \bar{\zeta}_{5,3}, \zeta_{9}, \bar{\zeta}_{7,3}, \zeta_{11}, \bar{\zeta}_{5,3,3}, \bar{\zeta}_{9,3}, \zeta_{13}, \bar{\zeta}_{5,5,3}, \bar{\zeta}_{7,3,3}, \bar{\zeta}_{6,4,1,1}
$$

The authors of [22] have published analytic results for a large collection of finite pintegrals. At 7 loops the collection contains 369 7-loop finite p-integrals which depends on single and multiple zeta values only. ${ }^{10}$

We have successfully checked the following.

1. All 369 p-integrals stop to depend on $\pi$ after being rewritten in terms of the proper generators (6.22) provided all terms proportional to $\zeta_{12}$ are set to zero by hand.

2. The disappearance of $\pi$-dependence in the above point holds not only for all terms with weight less or equal 11 but also for all (rather numerous) terms with the transcendental weight 12 and 13.

3. In fact, the database [22] contains also many finite p-integrals with loop number 8 . Some of them depend on multiple zeta values only. If one discards in these integrals all contributions with the transcendental weight strictly larger than 13 then there will remain exactly two non-vanishing integrals. After rewriting the survivors in terms of generators (6.22) and setting $\zeta_{12}$ to zero they also cease to depend on $\pi$ !

\footnotetext{
${ }^{10}$ We do not count uninteresting for our discussion cases of p-integrals depending only on single (odd) zetas, as the latter do not depend on $\pi$ at all neither in original nor in hatted forms in the limit of $\epsilon \rightarrow 0$.
} 


\section{Conclusion}

Using as input data essentially only deep $\epsilon$ expansions of the 4-loop master integrals [18] we have extended the hatted representation of 4-loop p-integrals of work [17] to the 5-, 6and 7-loop families of p-integrals. At 5-loop level we successfully reproduced the results of [20] which had been obtained by a direct calculation of a rather large subset of 5-loop master p-integrals.

We have derived a set of generic model-independent predictions for $\pi$-dependent terms of RG-functions at 7 and 8 loops (at the latter case only for terms with weight less or equal to 11). All available 7- and 8-loop results are in agreement with our predictions.

Our results demonstrate a remarkable and somewhat mysterious (at least for us) connection between $\epsilon$-expansions of the 4-loop p-integrals and $D=4$ values of 5 -, 6 - and 7-loop finite p-integrals. Indeed, dealing only with 4-loop p-integrals we have been able to get some non-trivial information about 5-, 6- and 7-loop p-integrals. More precisely, we have found a set of proper transcendental generators which form a $\pi$-free basis for every known 5-,6, and 7-loop p-integrals provided that (i) the latter is expressible only in terms of multiple zeta values and (ii) all terms (if any) proportional to $\zeta_{12}$ are discarded.

It would be interesting to see what new information can be extracted from expanding 4-loop master p-integrals to even higher orders in $\epsilon$.

\section{Acknowledgments}

We are very grateful to V. Smirnov for providing us with extension of the results of [18] to the transcendentality weight thirteen.

We thank J. Gracey for his help in extracting 6-, 7- and 8-loop terms from generic results of [30-32] and to O. Schnetz for providing us with his results for the 7-loop ADs in the $O(n) \phi^{4}$ model.

The work of P.A. Baikov is supported in part by the grant RFBR 17-02-00175A of the Russian Foundation for Basic Research. The work by K.G. Chetykin was supported by the German Federal Ministry for Education and Research BMBF through Grant No. 05H15GUCC1 and by the Deutsche Forschungsgemeinschaft through CH 1479/2-1.

Open Access. This article is distributed under the terms of the Creative Commons Attribution License (CC-BY 4.0), which permits any use, distribution and reproduction in any medium, provided the original author(s) and source are credited.

\section{References}

[1] P.A. Baikov and K.G. Chetyrkin, The structure of generic anomalous dimensions and no- $\pi$ theorem for massless propagators, JHEP 06 (2018) 141 [arXiv: 1804.10088] [INSPIRE].

[2] O. Schnetz, Numbers and Functions in Quantum Field Theory, Phys. Rev. D 97 (2018) 085018 [arXiv: 1606.08598] [INSPIRE].

[3] J.A. Gracey, Large $N_{f}$ quantum field theory, Int. J. Mod. Phys. A 33 (2019) 1830032 [arXiv: 1812.05368] [INSPIRE]. 
[4] S.G. Gorishnii, A.L. Kataev and S.A. Larin, The $O\left(\alpha_{s}^{3}\right)$-corrections to $\sigma_{\text {tot }}\left(e^{+} e^{-} \rightarrow\right.$ hadrons $)$ and $\Gamma\left(\tau^{-} \rightarrow \nu_{\tau}+\right.$ hadrons) in QCD, Phys. Lett. B 259 (1991) 144 [INSPIRE].

[5] R. Ayoub, Euler and the zeta function, Am. Math. Mon. 81 (1974) 1067.

[6] P.A. Baikov, K.G. Chetyrkin and J.H. Kühn, Adler Function, Bjorken Sum Rule, and the Crewther Relation to Order $\alpha_{s}^{4}$ in a General Gauge Theory, Phys. Rev. Lett. 104 (2010) 132004 [arXiv: 1001.3606] [INSPIRE].

[7] P.A. Baikov, K.G. Chetyrkin and J.H. Kühn, Five-loop fermion anomalous dimension for a general gauge group from four-loop massless propagators, JHEP 04 (2017) 119 [arXiv: 1702.01458] [INSPIRE].

[8] K.G. Chetyrkin, A.L. Kataev and F.V. Tkachov, New Approach to Evaluation of Multiloop Feynman Integrals: The Gegenbauer Polynomial x Space Technique, Nucl. Phys. B 174 (1980) 345 [INSPIRE].

[9] M. Jamin and R. Miravitllas, Absence of even-integer $\zeta$-function values in Euclidean physical quantities in QCD, Phys. Lett. B 779 (2018) 452 [arXiv:1711.00787] [InSPIRE].

[10] J. Davies and A. Vogt, Absence of $\pi^{2}$ terms in physical anomalous dimensions in DIS: Verification and resulting predictions, Phys. Lett. B 776 (2018) 189 [arXiv:1711.05267] [INSPIRE].

[11] K.G. Chetyrkin, G. Falcioni, F. Herzog and J.A.M. Vermaseren, Five-loop renormalisation of QCD in covariant gauges, JHEP 10 (2017) 179 [arXiv:1709.08541] [INSPIRE].

[12] B. Ruijl, F. Herzog, T. Ueda, J.A.M. Vermaseren and A. Vogt, The $R^{*}$-operation and five-loop calculations, PoS (RADCOR2017)011 [arXiv:1801.06084] [INSPIRE].

[13] F. Herzog, S. Moch, B. Ruijl, T. Ueda, J.A.M. Vermaseren and A. Vogt, Five-loop contributions to low-N non-singlet anomalous dimensions in QCD, Phys. Lett. B 790 (2019) 436 [arXiv: 1812.11818] [INSPIRE].

[14] P.A. Baikov, K.G. Chetyrkin and J.H. Kühn, Five-Loop Running of the QCD coupling constant, Phys. Rev. Lett. 118 (2017) 082002 [arXiv:1606.08659] [INSPIRE].

[15] F. Herzog, B. Ruijl, T. Ueda, J.A.M. Vermaseren and A. Vogt, The five-loop $\beta$-function of Yang-Mills theory with fermions, JHEP 02 (2017) 090 [arXiv: 1701.01404] [INSPIRE].

[16] T. Luthe, A. Maier, P. Marquard and Y. Schröder, The five-loop Beta function for a general gauge group and anomalous dimensions beyond Feynman gauge, JHEP 10 (2017) 166 [arXiv: 1709.07718] [INSPIRE].

[17] P.A. Baikov and K.G. Chetyrkin, Four Loop Massless Propagators: An Algebraic Evaluation of All Master Integrals, Nucl. Phys. B 837 (2010) 186 [arXiv:1004.1153] [INSPIRE].

[18] R.N. Lee, A.V. Smirnov and V.A. Smirnov, Master Integrals for Four-Loop Massless Propagators up to Transcendentality Weight Twelve, Nucl. Phys. B 856 (2012) 95 [arXiv:1108.0732] [INSPIRE].

[19] E. Panzer, On the analytic computation of massless propagators in dimensional regularization, Nucl. Phys. B 874 (2013) 567 [arXiv: 1305.2161] [INSPIRE].

[20] A. Georgoudis, V. Goncalves, E. Panzer and R. Pereira, Five-loop massless propagator integrals, arXiv:1802.00803 [INSPIRE].

[21] F. Brown, Feynman amplitudes, coaction principle, and cosmic Galois group, Commun. Num. Theor. Phys. 11 (2017) 453 [arXiv:1512.06409] [INSPIRE]. 
[22] E. Panzer and O. Schnetz, The Galois coaction on $\phi^{4}$ periods, Commun. Num. Theor. Phys. 11 (2017) 657 [arXiv: 1603.04289] [INSPIRE].

[23] D.J. Broadhurst and D. Kreimer, Association of multiple zeta values with positive knots via feynman diagrams up to 9 loops, Phys. Lett. B 393 (1997) 403 [hep-th/9609128] [INSPIRE].

[24] D. Broadhurst, Multiple Zeta Values and Modular Forms in Quantum Field Theory, Springer Vienna, Vienna, Austria (2013), pp. 33-73.

[25] P.A. Baikov and K.G. Chetyrkin, No- $\pi$ Theorem for Euclidean Massless Correlators, PoS (LL2018) 008 [arXiv: 1808.00237] [INSPIRE].

[26] D.J. Broadhurst, Dimensionally continued multiloop gauge theory, hep-th/9909185 [INSPIRE].

[27] J. Blumlein, D.J. Broadhurst and J.A.M. Vermaseren, The Multiple Zeta Value Data Mine, Comput. Phys. Commun. 181 (2010) 582 [arXiv:0907.2557] [InSPIRE].

[28] D.J. Broadhurst and D. Kreimer, Knots and numbers in $\varphi^{4}$ theory to 7 loops and beyond, Int. J. Mod. Phys. C 6 (1995) 519 [hep-ph/9504352] [INSPIRE].

[29] E. Panzer, Feynman integrals and hyperlogarithms, Ph.D. thesis, Humboldt University, 2015, [arXiv: 1506. 07243] [INSPIRE].

[30] J.A. Gracey, The QCD Beta function at $\mathcal{O}\left(1 / N_{f}\right)$, Phys. Lett. B 373 (1996) 178 [hep-ph/9602214] [INSPIRE].

[31] M. Ciuchini, S.E. Derkachov, J.A. Gracey and A.N. Manashov, Quark mass anomalous dimension at $\mathcal{O}\left(1 / N_{f}^{2}\right)$ in $Q C D$, Phys. Lett. B 458 (1999) 117 [hep-ph/9903410] [InSPIRE].

[32] M. Ciuchini, S.E. Derkachov, J.A. Gracey and A.N. Manashov, Computation of quark mass anomalous dimension at $\mathcal{O}\left(1 / N_{f}^{2}\right)$ in quantum chromodynamics, Nucl. Phys. B 579 (2000) 56 [hep-ph/9912221] [INSPIRE].

[33] A.N. Vasiliev, Yu.M. Pismak and Yu.R. Khonkonen, Simple Method of Calculating the Critical Indices in the 1/N Expansion, Theor. Math. Phys. 46 (1981) 104 [INSPIRE].

[34] A.N. Vasiliev, Yu.M. Pismak and Yu.R. Khonkonen, $1 / N$ Expansion: Calculation of the Exponents $\eta$ and $\nu$ in the Order $1 / N^{2}$ for Arbitrary Number of Dimensions, Theor. Math. Phys. 47 (1981) 465 [INSPIRE].

[35] A.N. Vasiliev, Yu.M. Pismak and Yu.R. Khonkonen, $1 / N$ Expansion: Calculation Of the Exponent $\eta$ in the Order $1 / N^{3}$ by the Conformal Bootstrap Method, Theor. Math. Phys. 50 (1982) 127 [INSPIRE].

[36] D.J. Broadhurst, J.A. Gracey and D. Kreimer, Beyond the triangle and uniqueness relations: Nonzeta counterterms at large $N$ from positive knots, Z. Phys. C 75 (1997) 559 [hep-th/9607174] [INSPIRE].

[37] A.V. Kotikov and S. Teber, On the Landau-Khalatnikov-Fradkin transformation and the mystery of even $\zeta$-values in Euclidean massless correlators, arXiv:1906.10930 [INSPIRE]. 